\title{
Novel Universal Phenomena of Single Slit, Double Slit, Cross-double Slit and Triple Slit Experiments - Curved Patterns and Orientation-Dependence of Patterns
}

hui peng ( $\square$ davidpeng1749@gmail.com )

N/A https://orcid.org/0000-0002-1844-3163

\section{Research Article}

Keywords: double slit experiments, cross-double slit experiments, single slit experiment, triple slit experiments, interference pattern, wave interpretation, quantum mechanics, optics

Posted Date: July 16th, 2021

DOl: https://doi.org/10.21203/rs.3.rs-722731/v1

License: (c) (1) This work is licensed under a Creative Commons Attribution 4.0 International License. Read Full License 


\title{
Novel Universal Phenomena of Single Slit, Double Slit, Cross-double Slit and Triple Slit Experiments --- Curved Patterns and Orientation-Dependence of Patterns
}

\author{
Hui Peng \\ Email: davidpeng1749@gmail.com
}

\begin{abstract}
Young's double slit experiments express the mystery of quantum mechanics. To explore the mystery, varieties of the double slit and cross-double slit experiments were performed. In this article, we show novel universal phenomena: (1) the fringes of the interference patterns of the double slit/cross-double slit/triple slit experiments can be distributed along curves, and the curvatures depend on the orientations of the diaphragms; (2) the characteristics of the interference patterns of the double slit/cross-double slit/triple slit experiments depend on the orientation of the diaphragm, specifically, on which axis the diaphragm rotates around, the rotating angle and how to rotate, clockwise or counterclockwise; and (3) in the cross-double slit experiments, the interference patterns created by the tilt double slits tend to incline towards the axis that is perpendicular to the axis the diaphragm rotates around. The single slit experiments have the same phenomena that, due to the rotation of the diaphragm around different axis, either the patterns are curved or the patterns expanded. We show the novel universal phenomena of the single slit/double slit/cross-double slit/triple slit experiments, which may be utilized in the applications of, for example, the double slit.
\end{abstract}

Keywords: double slit experiments, cross-double slit experiments, single slit experiment, triple slit experiments, interference pattern, wave interpretation, quantum mechanics, optics

Declaration: the author declares that there is no interesting conflict.

\section{Content}

1. Introduction

2. Apparatus

3. Characteristics of Pattern Depending on Orientation of Diaphragm

3.1. Double Slit: Orientation-Dependence of Interference Pattern

3.1.1. Rotating Diaphragm Around Y-Axis: Interference Patterns Curved
(A) Discrete Rotation
(B) Continuous Rotation: Appendix-1 Video

\subsubsection{Rotating Diaphragm Around Z-Axis: Interference Pattern Varied}

(A) Derivation of Formular of Orientation-dependence of Interference Pattern 
(B) Rotating Diaphragm Around Z-Axis

3.1.3. Rotating Diaphragm Around X-axis: Rotation-Invariance

3.2. Cross-Double Slit: Orientation-Dependence of Interference Pattern

3.2.1. Rotating Diaphragm Around Y-Axis: Interference Patterns Curved and Varied

3.2.2. Rotating Diaphragm Around Z-Axis: Interference Patterns Varied

3.2.3. Rotating Diaphragm Around X-Axis: Rotation-Invariance

3.3. Triple Slit: Orientation-Dependence of Interference Pattern

3.3.1. Rotating Diaphragm Around Y-Axis: Interference Patterns Curved

3.3.2. Rotating Diaphragm Around Z-Axis: Interference Pattern Varied

3.3.3. Rotating Diaphragm Around X-Axis: Rotation-Invariance

3.4. Single Slit: Orientation-Dependence of Pattern

3.4.1. Rotating Diaphragm Around Y-Axis: Pattern Curved

3.4.2. Rotating Diaphragm Around Z-Axis: Pattern Varied

3.4.3. Rotating Diaphragm Around X-Axis: Rotation-Invariance

4. Summary

4.1. Comparing Experiments with Same Diaphragm and Rotating Around Different Axis

4.2. Comparing Experiments with Different Diaphragms and Rotating Around Same Axis Appendix

A-1 Video: Evolution of Curved Interference Pattern of Double Slit Experiment

A-2 Potential Applications of Universal Phenomena

\section{Introduction}

Young's double slit experiment was first performed in 1801 [1,2], which, 100 years later, led to wave-particle duality. Feynman called the double slit experiment "a phenomenon which is impossible [...] to explain in any classical way, and which has in it the heart of quantum mechanics. In reality, it contains the only mystery [of quantum mechanics]." [3]. Moreover, the nature of photons truly puzzled Einstein. He wrote to M. Besso: "All these 50 years of conscious brooding have brought me no nearer to the answer to the question: What are light quanta?" [4].

On the other hand, in the double slit experiments, the optical characteristics of the interference pattern is described by three factors, wave length, the spacing between two slits and distance between the double slit and screen, as described by equation, $y_{\text {const }}=m \frac{\lambda}{d} L$.

Note that (1) there is only one factor related to the parameter of the diaphragm of the double slit, i.e., the spacing d; (2) the fringes of the interference pattern distribute along a straight line. 
We raise a question whether there are other factors that are related with the diaphragm and affect the characteristics of the interference pattern? To answer this question, we emphasize that in the standard double slit experiments, the light beam is perpendicular to the plane of the diaphragm of the double slit.

We are interested in how the characteristics of the interference patterns vary with different orientations of the diaphragm of double slit/cross-double slit [5,6]. In this article, we study, by rotating the diaphragms around the $\mathrm{x}$-axis, $\mathrm{y}$-axis, and $\mathrm{z}$-axis, respectively, the orientation-dependence of the characteristics of the interference patterns of the double slit/cross-double slit/triple slit experiments and the orientation-dependence of the characteristics of the patterns of the single slit experiments. The orientation of the diaphragm contains three factors: the axis the diaphragm rotating around, the rotation direction, i.e., clockwise or counterclockwise, the angle of rotation. Those factors related the defined coordinate with $\mathrm{X}$-axis, $\mathrm{Y}$-axis and Z-axis.

We show the novel universal phenomena that the pattern/interference patterns of single slit, double slit, cross-double slit and triple slit experiments can be along curved line and the characteristics of patterns/interference patterns are determined by the orientations of the diaphragms.

The novel universal phenomena, for example, the expansion of the patterns/interference patterns, may be utilized in the applications of, for example, the double slit.

\section{Apparatus}

The experiments utilize a laser source, the diaphragms of the double slit (Figure 1a) and cross-double slit (Figure 1b), a protractor (Figure 1c) and a screen/detector.

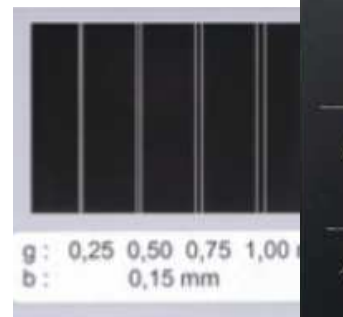

(a)

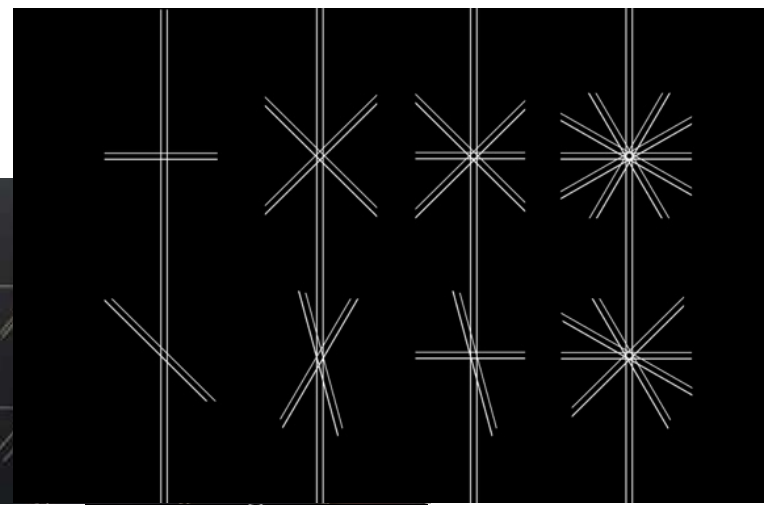

(b)

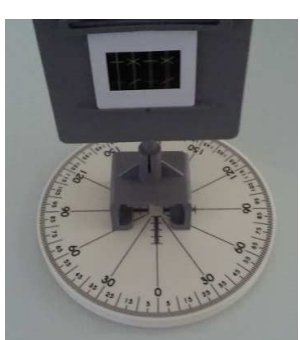

(c)

Figure 1 Diaphragms and protractor

We define: $\mathrm{X}$-axis is along the normal vector of the plane of the diaphragms; Y-axis is in the plane and perpendicular to either single slit or double slit or one double slit of cross-double slit or triple slit; Z-axis is aligned with either single slit or double slit or one double slit of cross-double slit or triple slit. 


\section{Characteristics of Patterns Depending on Orientation of Diaphragm}

3.1. Double Slit: Orientation-Dependence of Interference Pattern

\subsubsection{Rotating Diaphragm Around Y-Axis: Interference patterns Curved}

\section{(A) Discrete Rotation}

The schematic of the experimental setup for the double slit experiments is shown in Figure 2.

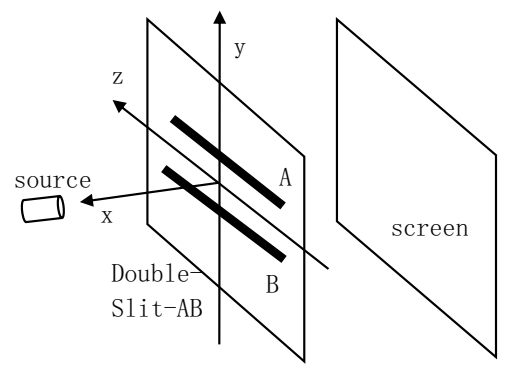

Figure 2 Schematic drawing of apparatus

The double-slit-AB is in the $\mathrm{y}-\mathrm{z}$ plane, slit $\mathrm{A}$ and slit $\mathrm{B}$ are along the $\mathrm{z}$-axis, its normal vector is along the $\mathrm{x}$-axis and points to source, the spacing between slits A and B is " $\mathrm{d}$ ", and photons travel along the negative $\mathrm{x}$ direction. Its interference pattern is shown in Figure 3a.

The standard optical equation, $y_{\text {const }}=m \frac{\lambda}{d} L$, is derived for a special situation that the source is on the normal vector of the plane of the double slit. We study how the characteristics of interference patterns vary with the different orientations of the diaphragm of the double slit.

Experiment-1: rotating the double slit-AB clockwise around Y-axis with different discrete angles, $45^{\circ}$, $60^{\circ}$ and $75^{\circ}$. The original orientation corresponds to $0^{0}$ rotation.

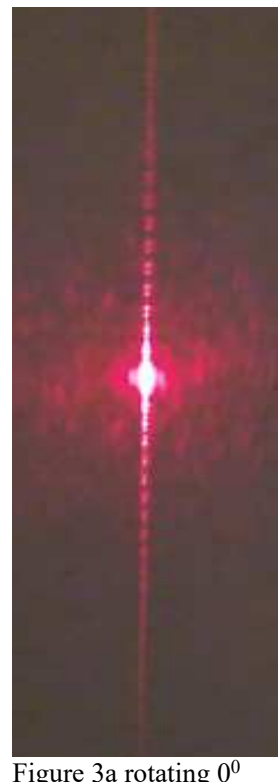

Figure 3 a rotating $0^{0}$

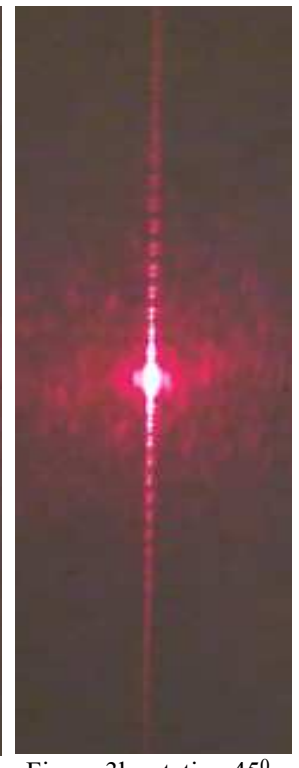

Figure 3 b rotating $45^{0}$

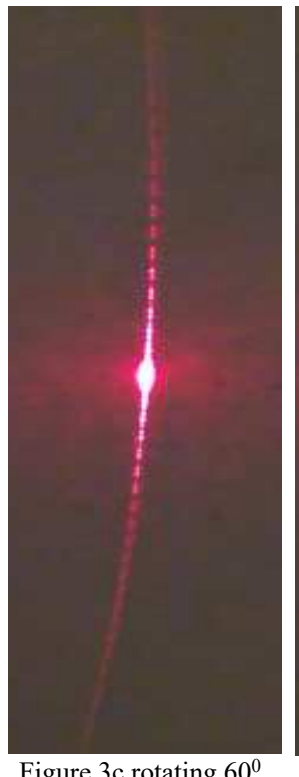

Figure $3 \mathrm{c}$ rotating $60^{0}$

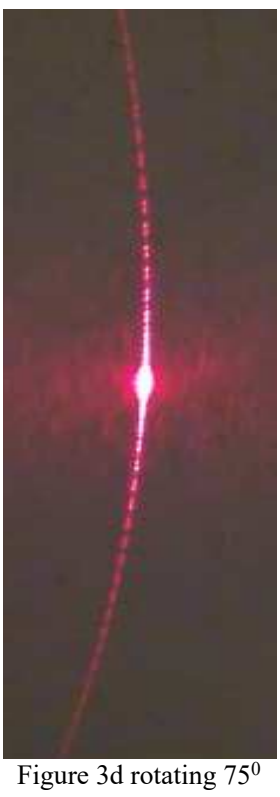

Figure $3 \mathrm{~d}$ rotating $75^{0}$

Observation (Figure 3): the interference patterns curved towards the left side. The larger the rotation angle, the smaller curvature of the interference patterns.

Experiment-2: rotating the double slit counterclockwise with different discrete angles (Figure 4): 


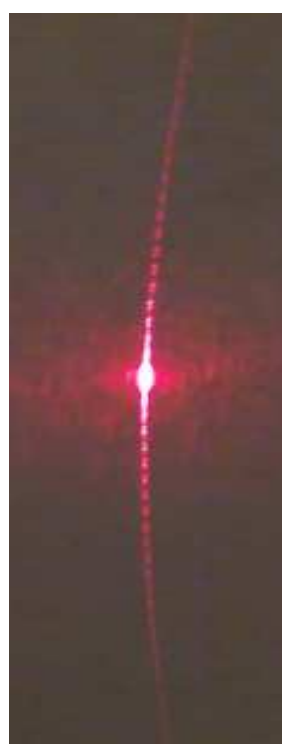

Figure 4 a rotating $45^{0}$

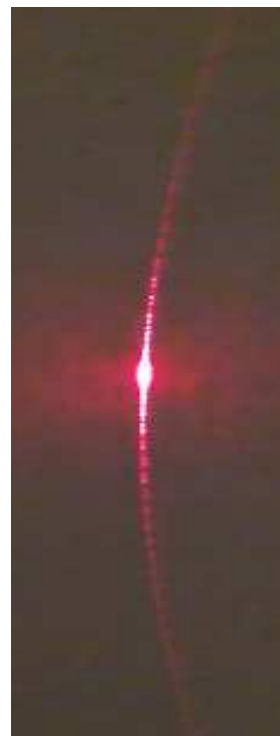

Figure $4 \mathrm{~b}$ rotating $60^{\circ}$

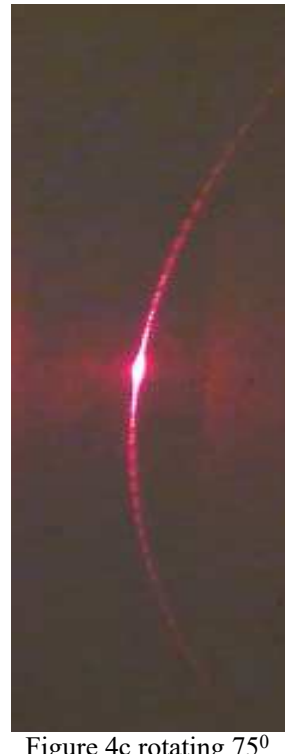

Figure $4 \mathrm{c}$ rotating $75^{0}$

Observation: the interference patterns curved towards the right side that is opposite to that of the curved interference pattern created by rotating the double slit clockwise.

Discussion: in the regular double slit experiment, photons only need to know one factor: whether there is a double slit or not. Now we show that photons also need to know more factors: (1) which axis the diaphragm rotating around; (2) the direction of rotation, i.e., clockwise or counterclockwise; (3) the angle of the rotation.

\section{(B) Continuous Rotation: Appendix-1 Video}

Let us place the double slit at the position that the double slit rotates $75^{0}$ from the original orientation counterclockwise. Turning on the laser source, we observed the curved interference patten. Then rotating the double slit clockwise continuously and the curved pattern continuously change, the curvature of the interference pattern becomes larger and larger. We reach a position that the laser light is perpendicular to the plane of the double slit, which we referred as the original position, and at that position, the curved interference pattern becomes the regular straight interference pattern. Then continuously rotating the double slit clockwise. The interference pattern starts to curve again but to the opposite direction. See the attached Appendix-1 Video.

\subsubsection{Rotating Diaphragm Around Z-axis: Interference Pattern Varied}

Let us derive the Orientation-dependence Formular first. Then we use the formular to describe the experiment and show that the experiments support the derived formular.

\section{(A) Derivation of Formular of Orientation-dependence of Interference Pattern}

The schematic drawing (not to scale) is the following. 


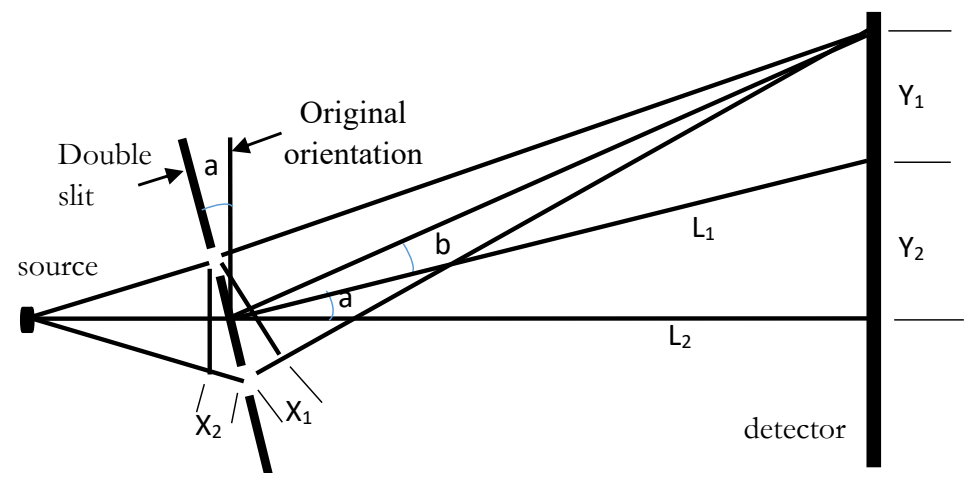

Figure 5 Schematic drawing for derivation of orientation-dependence of interference pattern

The path difference between two waves passing through two slits respectively is $\left(x_{1}+x_{2}\right)$. The requirement of the interference of two waves is that the path difference satisfies the following relation,

$$
x_{1}+x_{2}=m \lambda,
$$

where, $x_{1}$ is the path difference after passing the double slit, while $x_{2}$ is the path difference before arriving the double slit,

$$
\begin{aligned}
& x_{1} \approx d \sin b, \\
& x_{2} \approx d \sin a, \\
& \sin a=\frac{y_{2}}{L_{1}}=\frac{y_{2}}{\sqrt{L_{2}^{2}+y_{2}^{2}}}=\frac{y_{2}}{L_{2}} \frac{1}{\sqrt{1+y_{2}^{2} / L_{2}^{2}}}, \\
& \sin b \approx \tan b \approx \frac{y_{1}}{L_{1}} .
\end{aligned}
$$

Substituting Eq. (2), Eq. (3), Eq. (4) and Eq. (5) into Eq. (1), we obtain the equation of the orientation-dependence of the constructive/destructive interference pattern of the double slit,

$$
\begin{aligned}
& y \equiv y_{1}+y_{2}=\frac{m \lambda}{d} L_{2} \sqrt{1+(\tan a)^{2}}, \\
& y=\left(m+\frac{1}{2}\right) \frac{\lambda}{d} L_{2} \sqrt{1+(\tan a)^{2}},
\end{aligned}
$$

where, $(\tan a)^{2}=\frac{y_{2}^{2}}{L_{2}^{2}}, " \mathrm{y}$ " is the position of a bright/dark fringe from the zeroth-order fringe. In the standard derivation of the formular of the interference pattern, there is no $x_{2}$.

\section{(B) Rotating Diaphragm Around Z-axis}

The experiments show the rotating-angle-dependence of the interference patterns visually.

\section{Experiment-3:}

(1) The diaphragm at the original orientation and its interference pattern (Figure 6).
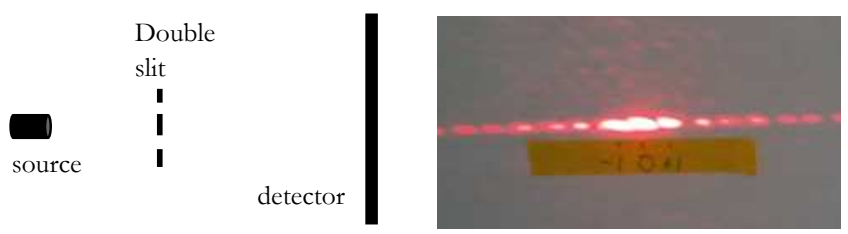

Figure 6 The diaphragm at original orientation and its pattern

Equation (6) gives $y=\frac{m \lambda}{d} L_{2}$, which is the normal situation. 
(2) The diaphragm rotates $30^{\circ}$ from the original orientation (Figure 7).
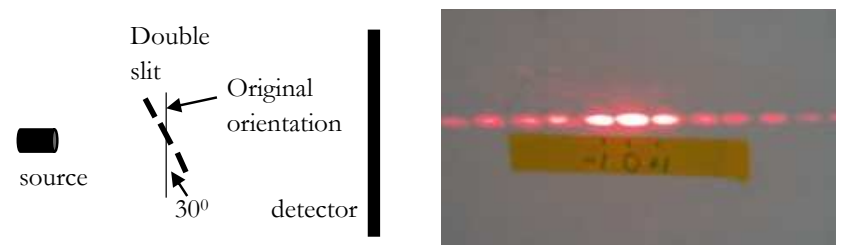

Figure 7 The diaphragm rotates $30^{\circ}$ and its pattern

Eq. (6) gives $y \approx 1.15 \frac{m \lambda}{d} L_{2}$.

(3) The diaphragm rotates $45^{0}$ from the original orientation (Figure 8).
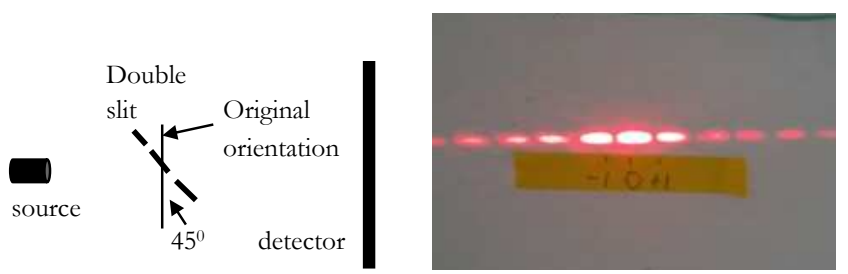

Figure 8 The diaphragm rotates $45^{0}$

Eq. (6) gives $y \approx 1.4 \frac{m \lambda}{d} L_{2}$.

(4) The diaphragm rotates $60^{\circ}$ from the original orientation (Figure 9).

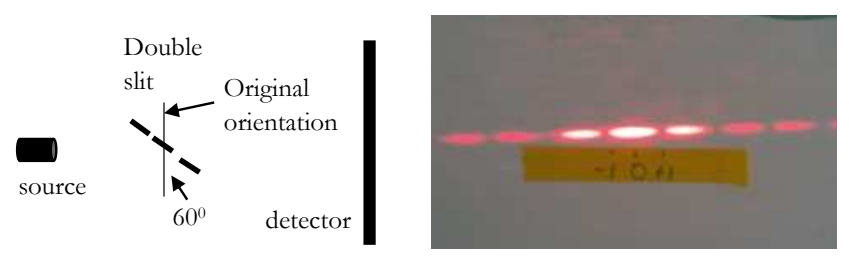

Figure 9 The diaphragm rotates $60^{\circ}$

Eq. (6) gives $y \approx 2 \frac{m \lambda}{d} L_{2}$.

(5) The diaphragm rotates $75^{0}$ from the original orientation (Figure 10).
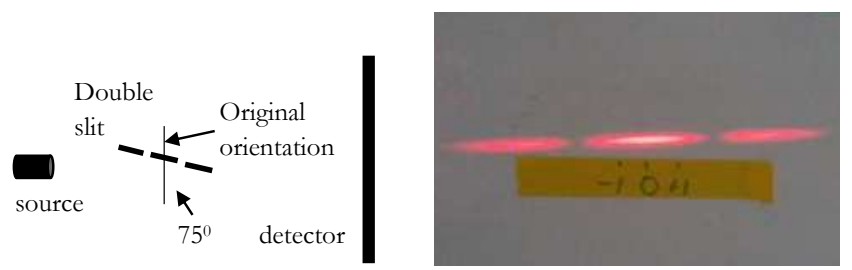

Figure 10 The diaphragm rotates 75 degrees

Eq. (6) gives $y \approx 3.86 \frac{m \lambda}{d} L_{2}$.

Conclusion: The experimental results support the derived formular (6). Figure 6 to figure 10 show the evolution of the interference pattern varying with angles of the double slit rotating around the z-axis. The larger the rotation angle, the larger expansion of the pattern, namely the distances between fringes are expanded. 


\subsubsection{Rotating Diaphragm Around $\mathrm{X}$-axis: Rotation-Invariance}

For the completeness of the study of the double slit experiments, let us rotate the diaphragm of the double slit around $\mathrm{X}$-axis, although it is trivial.

Experiment-4: Figure 11 shows the interference pattern without rotation. Figure 12 show the interference pattern after the diaphragm rotating 45 degrees.

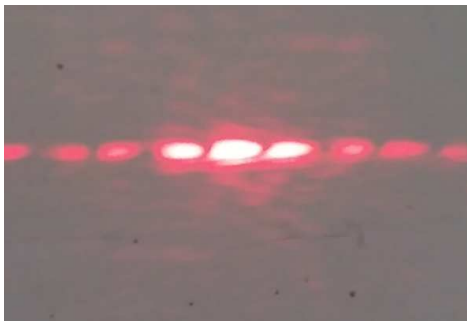

Figure 11 rotating $0^{0}$

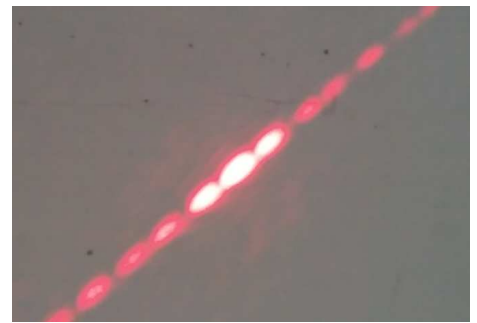

Figure 12 rotating $45^{0}$

Conclusion: the interference pattern does not vary; call it the rotation-invariance.

\subsection{Cross-Double Slit: Orientation-Dependence of Interference Pattern}

Now we study whether those novel phenomena of the double slit, shown in section 3.1, would happen to the cross-double slit. To show the orientation-dependence of the interference pattern of the cross-double slit, we use the tilt-cross-double slit, e.g., two double slits crossing at $15^{\circ}$ and $30^{\circ}$ to the vertical double slit respectively. We show that, beside the above phenomena of the double slit, the cross-double slit experiments indicate the novel phenomena that the patterns created by two tilt-double slits change the inclined angles. The cross-double-slit apparatus consists of source, tilt-cross-double-slit, protractor and screen. The laser source is on the normal vector of the diaphragm for the original orientation, rotating $0^{0}$. When the diaphragm rotates, the laser source stay.

\subsubsection{Rotating Diaphragm Around Y-axis: Interference Patter Curved and Varied}

Figure 13 shows the diaphragm rotating $0^{0}$ around $\mathrm{Y}$-axis, referred as the original orientation. The laser source is on the normal vector of the $\mathrm{Y}-\mathrm{Z}$ plane, the $\mathrm{X}$-axis. Its interference pattern shown on the right of Figure 13. The interference patterns are created independently. The angles between the interference pattens are the same as that of between the slits. The screen is 10 feet away.
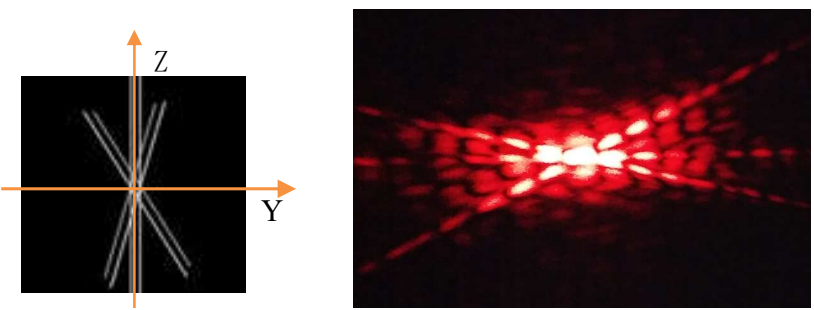

Figure 13 Original Orientation: Rotating $0^{0}$ 


\section{Experiment-5 (Figure 14, 15, 16).}

Let us rotate the diaphragm $60^{\circ}$ and $75^{\circ}$ respectively around $Y$-axis clockwise. The interference patterns are shown in Figure 15 and Figure 16. Figure 14 shows the original pattern.

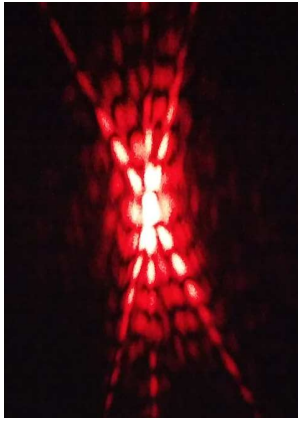

Figure 14 Original

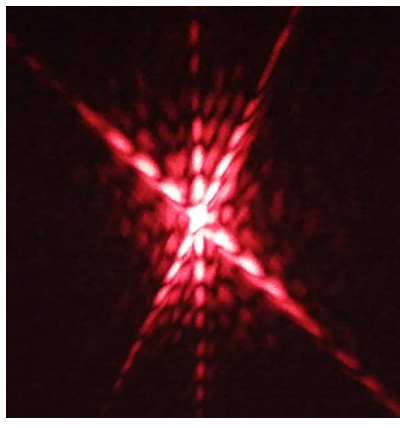

Figure 15 Rotating $60^{\circ}$

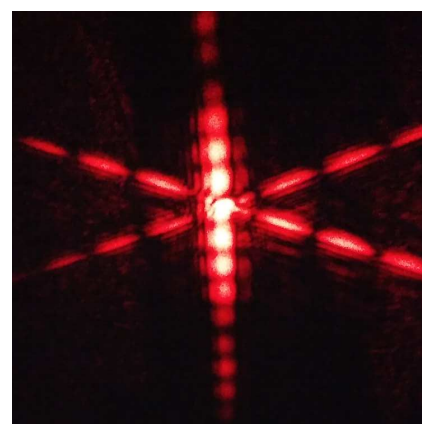

Figure 16 Rotating $75^{0}$

Observation: (1) Figure 15 and Figure 16 show: (1) the vertical patter curved towards the left; (2) the distances between fringes of tilt interference pattens become longer; (3) the angles between the tilt interference patterns and the vertical interference patten become larger.

Experiment-6: Rotating the diaphragm $60^{\circ}$ and $75^{\circ}$ respectively around Y-axis counterclockwise. The patterns are shown in Figure 17 and Figure 18.

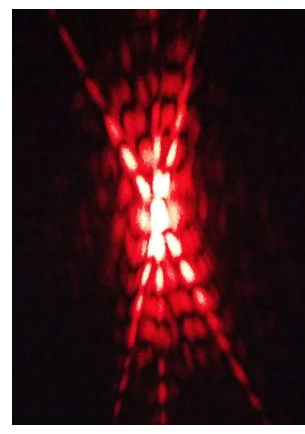

Figure 14

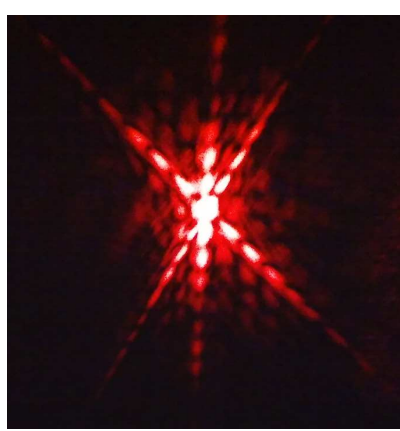

Figure 17 Rotating $60^{\circ}$

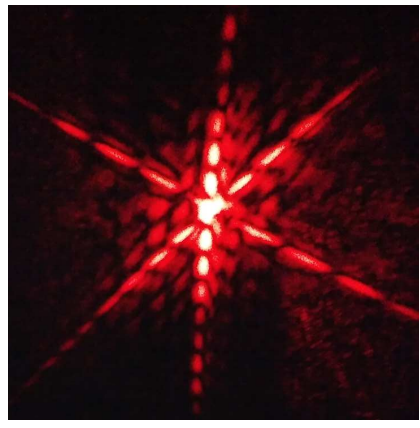

Figure 18 rotating $75^{0}$

Observation: (1) Figure 17 and Figure 18 show: (1) the vertical patter curved towards the right; (2) the distances between fringes of tilt interference pattens become longer; (3) the angles between the tilt interference patterns and the vertical interference patten become larger.

Discussion: (1) Figure 14 to Figure 18 show: (1) the larger the rotating angle of the diaphragm, the larger the angles between the interference patterns created by the tilt double slits and the horizontal interference pattern created by the vertical double slit; (2) The interference patterns created by the tilt double slits tend to incline closer to Z-axis; (3) the larger the rotating angle of the diaphragm, the larger the distances between the fringes of the interference patterns created by the tilt double slits; (4) the interference patterns created by the vertical double slit curved towards opposite directions. 
We show that the characteristics of the interference patterns of the cross-double slit depend on the orientation of the diaphragms. This experiment also shows that photons' behavior also depend on which slit they passing through.

\subsubsection{Rotating Diaphragm Around Z-axis: Interference Pattern Varied}

Now we show the orientation-dependence of the interference pattern of the cross-double slit, especially the novel phenomena that the patterns created by two tilt-double slits incline towards the horizontal pattern. The screen is 20 feet away

Experiment-7: First, the diaphragm is at the original orientation, i.e., rotates $0^{0}$ (Figure 13).
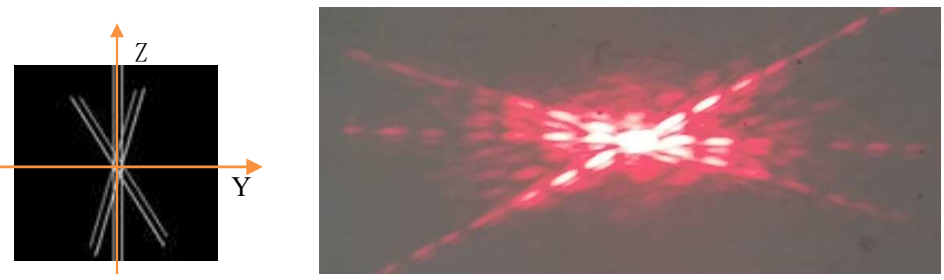

Figure 13 Rotating $0^{0}$

Experimental Step-1 (Figure 19): the diaphragm rotates $60^{\circ}$ around Z-axis.

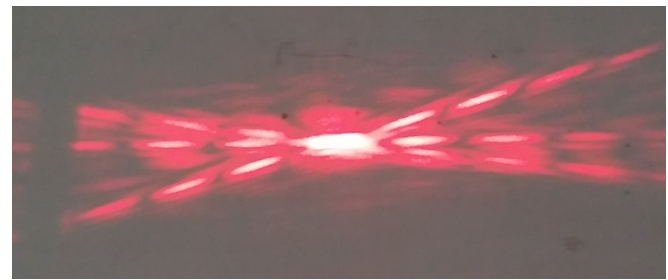

Figure 19 Rotating $60^{0}$

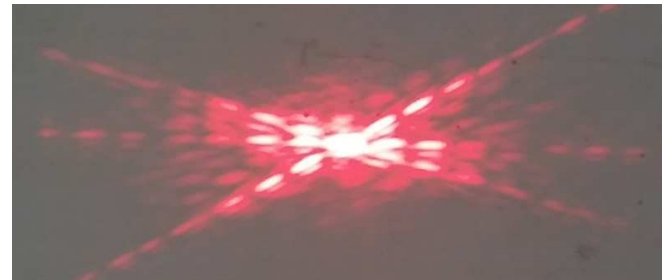

Figure 13 Rotating $0^{0}$

Observation (Figure 19): the angles between two interference patterns are smaller than that shown in Figure 13. The distances between two fringes of the same patten are larger than that in Figure 13.

Experimental step-2 (Figure 20): the diaphragm rotates $75^{\circ}$ around Z-axis

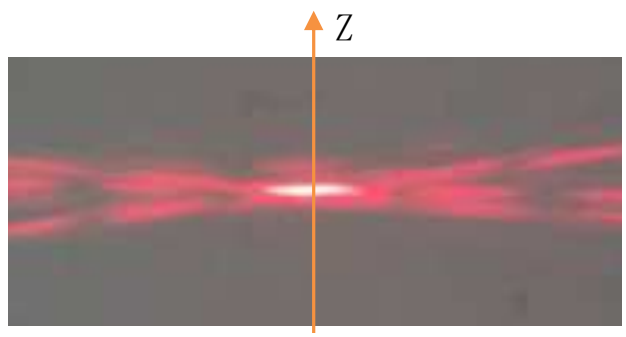

Figure 20 Rotating $75^{0}$

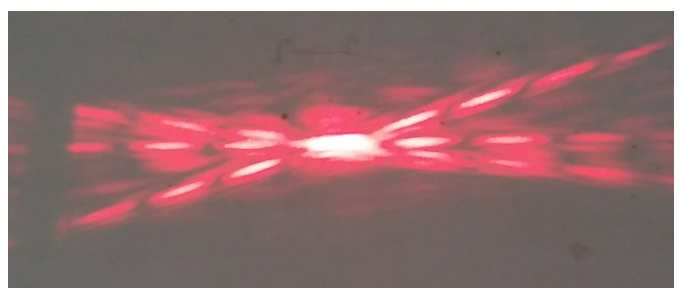

Figure 19 Rotating $60^{0}$

Observation (Figure 20): the angles between two interference patterns are smaller than that shown in Figure 19. The distances between two fringes are larger than that shown in Figure 17.

Conclusion: The interference patterns created by two tilt double slits tend to incline towards Y-axis. The characteristics of the interference patterns vary with the rotating angles. Namely, the larger the 
rotation angle, the larger the expansion of pattern, and the smaller the angles between the interference patterns.

\subsubsection{Rotating Diaphragm Around $\mathrm{X}$-axis: Rotation-Invariance}

$\mathrm{X}$-axis is along the Normal Vector.

Experiment-8: rotating the diaphragm (Figure 13) around X-axis $45^{\circ}$.

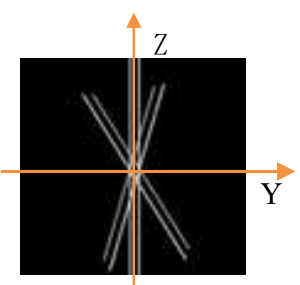

Figure 13

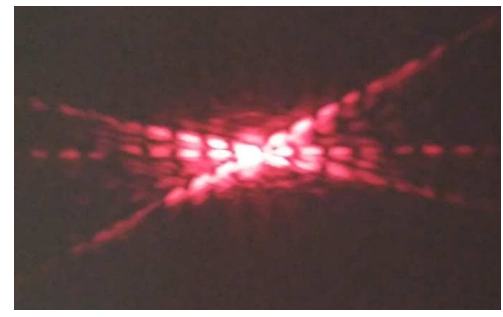

Figure 13 Original

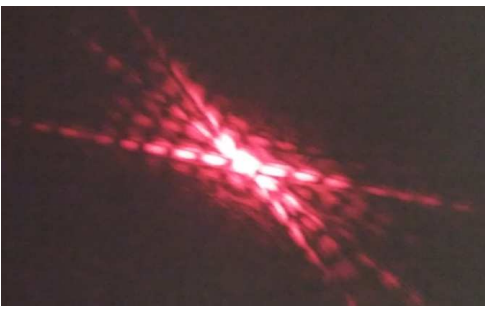

Figure 21 Rotate around A-axis

Observation: Rotating the diaphragm $45^{\circ}$, the interference pattern rotating the same angle (Figure 21), which shows the rotation-invariance around $\mathrm{X}$-axis.

\subsection{Triple Slit: Orientation-Dependence of Interference Pattern}

\subsubsection{Rotating Diaphragm Around Y-axis: Interference Pattern Curved}

The diaphragm of the triple slit is shown in Figure 22.

Experiment-9: We rotate the diaphragm counterclockwise and clockwise $75^{\circ}$ respectively.

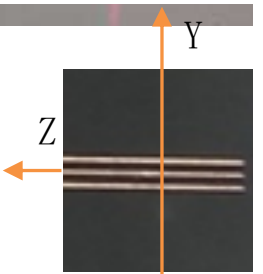

Figure 22

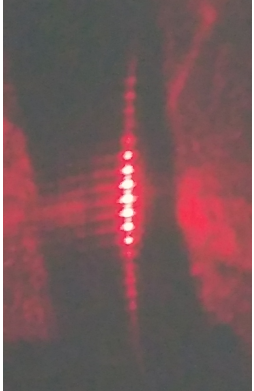

Figure 23

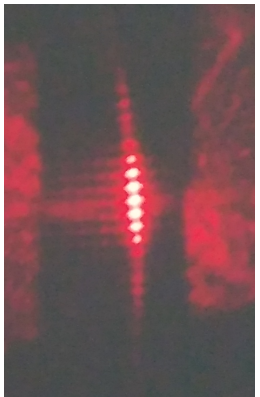

Figure 24

Observation: Figure 23 shows the interference pattern curved towards the right attributed to the counterclockwise rotating of the diaphragm. Figure 24 shows the interference pattern curved towards the left attributed to the clockwise rotating of the diaphragm.

\subsubsection{Rotating Diaphragm Around Z-axis: Interference Pattern Varied}

Figure 25 show the diaphragm of triple slit and its interference pattern. The screen is at 20 feet. 

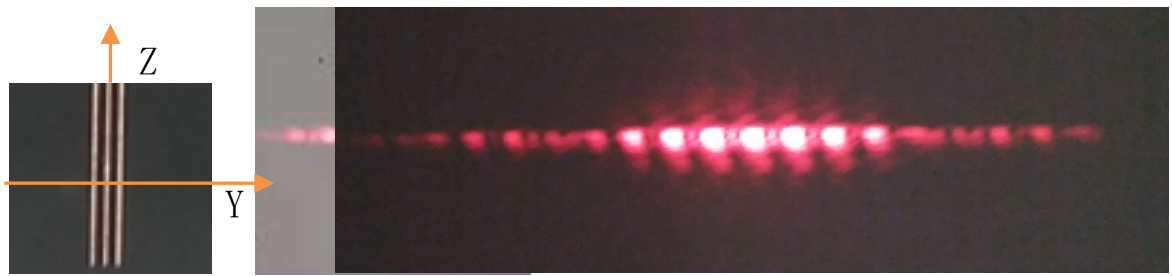

Figure 25 Triple slit and pattern: rotating $0^{0}$

Experiment-10: Rotating the diaphragm of triple slit $60^{\circ}$ and $75^{\circ}$ around Z-axis respectively.

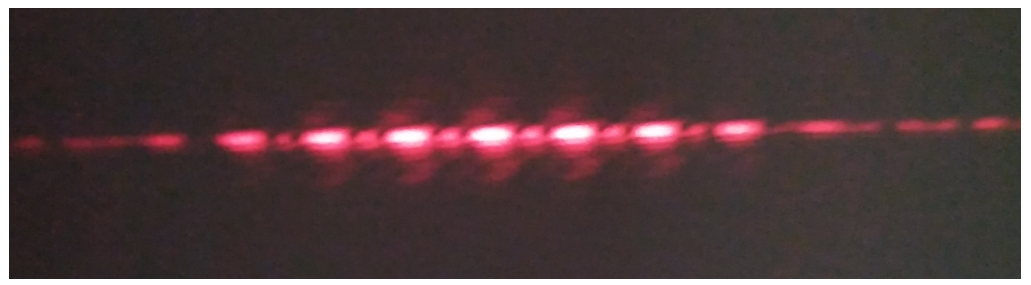

Figure 26 Rotating $60^{\circ}$

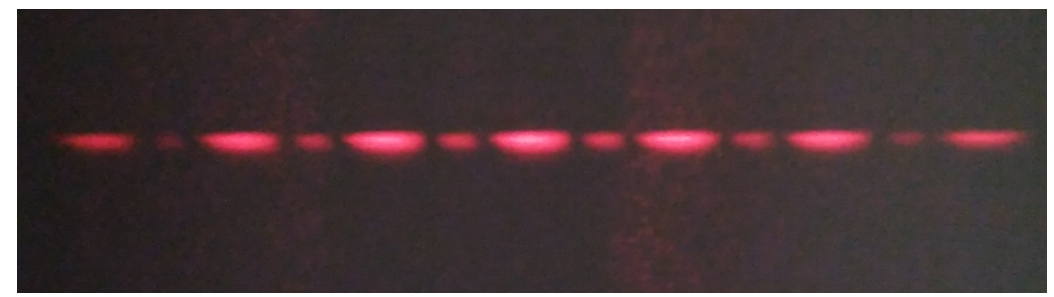

Figure 27 rotating $75^{0}$

Observation (Figure 26 and 27): The triple slit rotates around Z-axis. The larger the rotation angle, the larger the expansion of the pattern, namely the distances between fringes are expanded.

\subsubsection{Rotating Diaphragm Around $\mathbf{X}$-axis: Rotation-Invariant}

Experiment-11: rotating the triple slit $45^{\circ}$ around X-axis (Figure 28).

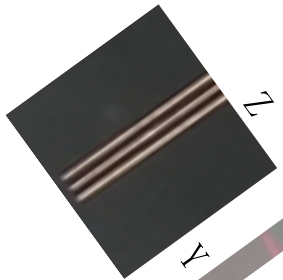

(a)

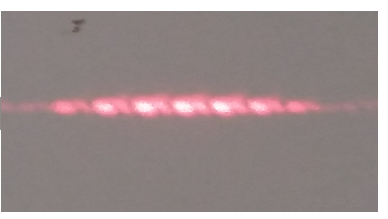

(b)

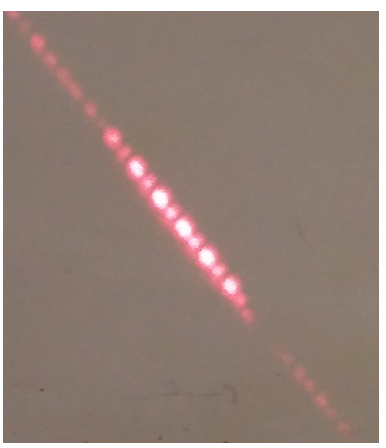

(c)

Figure 28 Rotating around $\mathrm{X}$-axis

Observation/conclusion: the original pattern (Figure 28b) does not change, but rotates the same angle

(Figure 28c). The interference pattern of the triple slit have the rotation symmetry around X-axis. 


\subsection{Single Slit: Orientation-Dependence of Pattern}

We have shown the orientation-dependence of the interference patterns of the double slit, cross-double slit and triple slit. It is interesting to study the pattern created by the single slit, Figure 29.
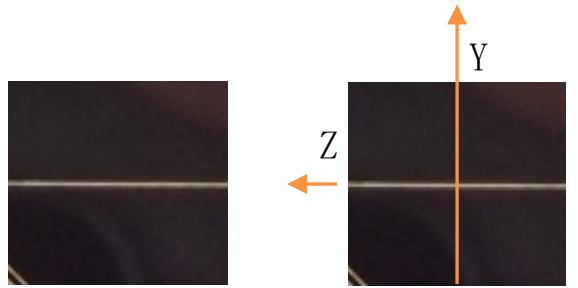

Figure 29 Single slit

\subsubsection{Rotating Diaphragm Around Y-axis: Pattern Curved}

Experiment-12 (Figures 30, 31 and 32): Rotating the diaphragm of the single slit around Y-axis.

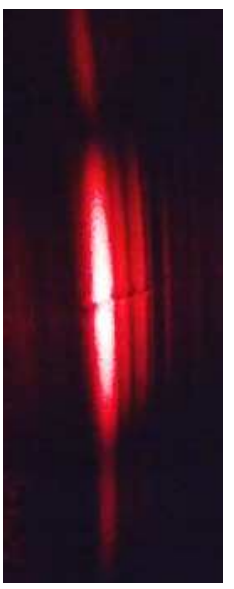

Figure 31

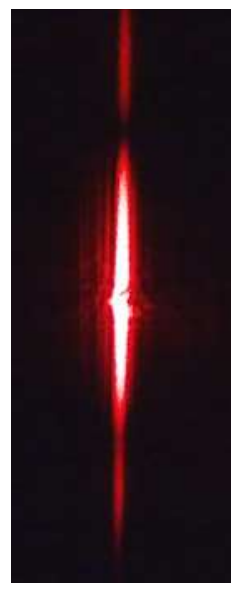

Figure 30

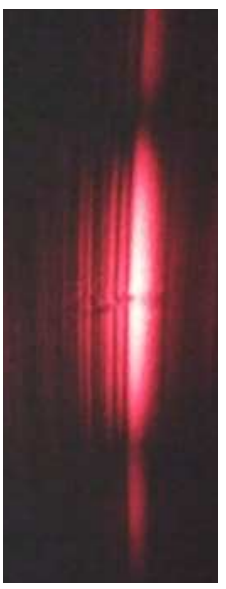

Figure 32

Observation: At the original orientation, $0^{0}$ rotation, Figure 30 shows its pattern. Then rotating $75^{0}$ clockwise and counterclockwise around Y-axis respectively, the created patterns are curved towards the left and the right as shown in Figure 31 and Figure 32 respectively. .

\subsubsection{Rotating Diaphragm Around Z-axis: Pattern Varied}

At the original orientation the pattern is the standard one (Figure 33).
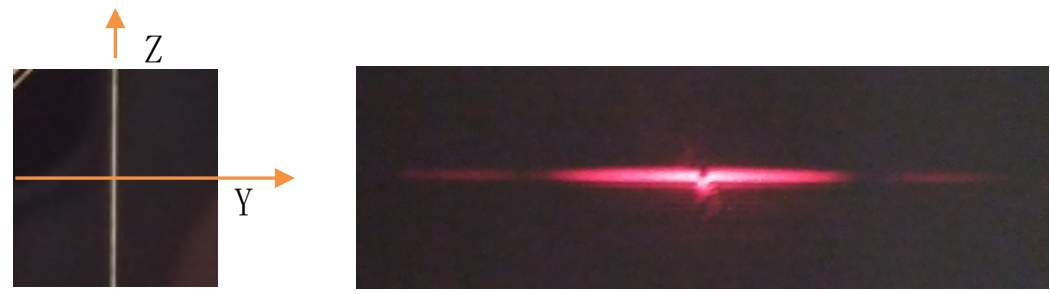

Figure 33 Single slit and its pattern

Experiment-13: Rotating the single slit around Z-axis $60^{\circ}$ and $75^{\circ}$, respectively. 
Figure 34 Rotating $60^{\circ}$

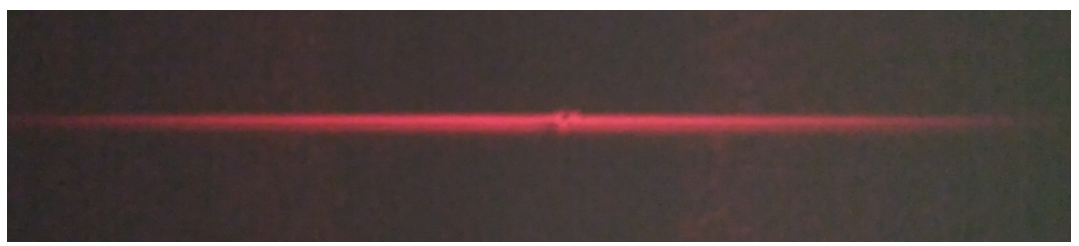

Figure 35 Rotating $75^{0}$

Observation: the pattern expanded (Figure 34,35). The larger the angle rotated, the longer the expansion of the pattern. Either clockwise or counterclockwise rotation give the same pattern.

Discussion: the single slit experiments show that its patterns can be curved and expanded by rotating the diaphragm, which is the similar to that of the interference patterns.

\subsubsection{Rotating Diaphragm Around $\mathrm{X}$-axis: Rotation-Invariant}

Experiment-14: The single slit has the rotation-invariance around X-axis (Figure 36).
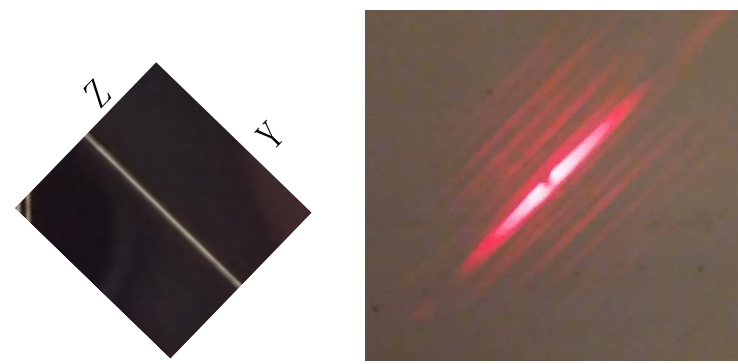

Figure 36 Rotating $45^{0}$ around $\mathrm{X}$-axis

\section{Summary}

To summarize, let us compare the patterns/interference patterns of the single slit, double slit, cross-double slit and triple slit experiments when the diaphragms rotating around $\mathrm{X}$-axis, $\mathrm{Y}$-axis and Z-axis, respectively.

We define: $\mathrm{X}$-axis is along the normal vector of the plane of the diaphragms; Y-axis is in the plane and perpendicular to either single slit or double slit or one double slit of cross-double slit or triple slit; Z-axis is aligned with either single slit or double slit or one double slit of cross-double slit or triple slit.

We compere those experiments in two perspectives.

First perspective: comparing the experiments with the same diaphragm and rotate around different axis, which show the orientation-dependence of the patterns; 
Second perspective: comparing the experiments with different diaphragm and rotate around the same axis, which show the similar phenomena for different kinds of diaphragms, we call it the "Universal phenomena".

\subsection{Comparing Experiments with Same Diaphragm Rotating Around Different Axis}

A) Double Slit: Rotating around X-axis, Y-axis, Z-axis

With the same diaphragm of double slit (Figure 2), the comparison of Figure 11 (the original interference pattern), Figure 12 (rotating around X-axis), Figure 4 and Figure 3 (rotating around Y-axis clockwise and counterclockwise) and Figure 10 (rotating around Z-axis) shows that which axis the diaphragm rotating around determine the photons' behavior/characteristics of interference pattern.

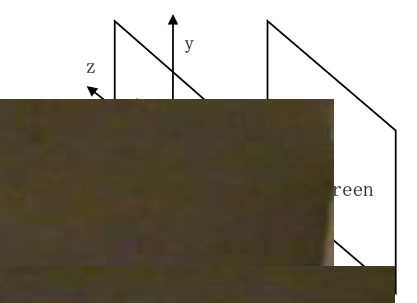

Figure 2

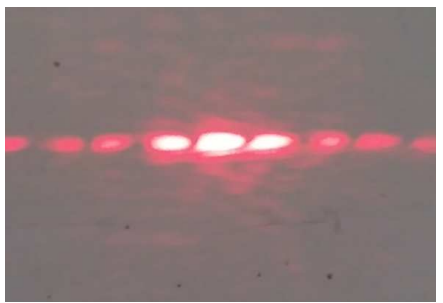

Original (Figure 11)

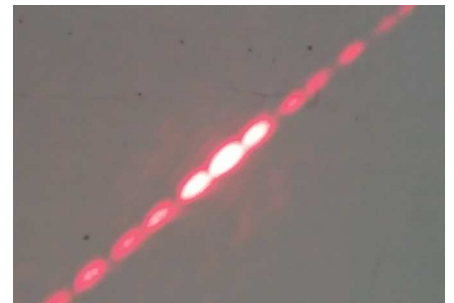

Around $\mathrm{X}$-axis (Figure 12)

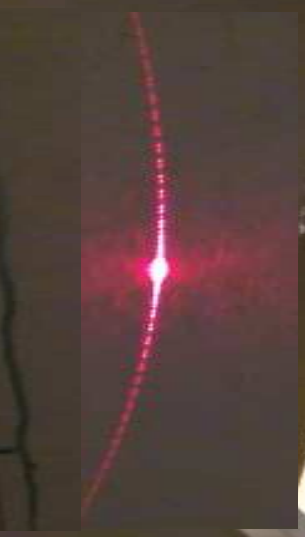

Around Y-axis (Fig. 3)

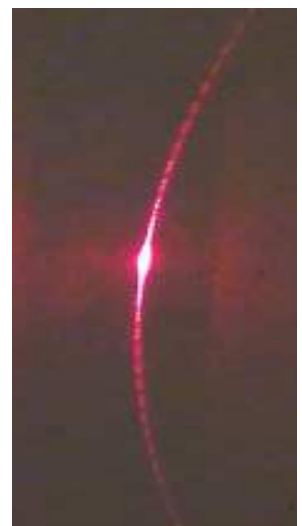

Around Y-axis (Fig. 4)

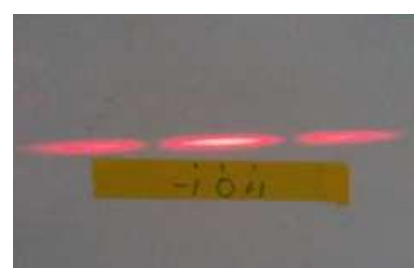

Around Z-axis (Fig. 10)

B) Cross-Double Slit: Rotating around X-axis, Y-axis, Z-axis

With the same diaphragm (Figure 13), comparing Figure 21 (rotating around X-axis), Figure 16/18 (rotating around Y-axis clockwise/counterclockwise) and Figure 19/20 (rotating around Z-axis), we conclude that which axis the diaphragm rotates around determines the photon's behavior and pattern. 


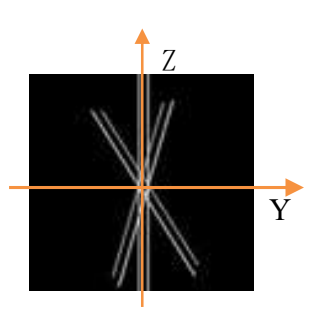

Figure 13

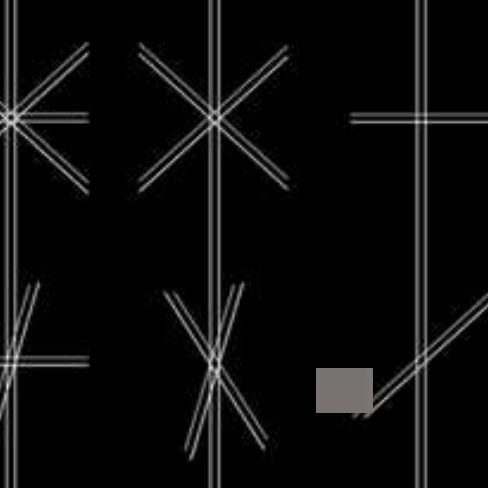

Around Y-axis (Figure 16)

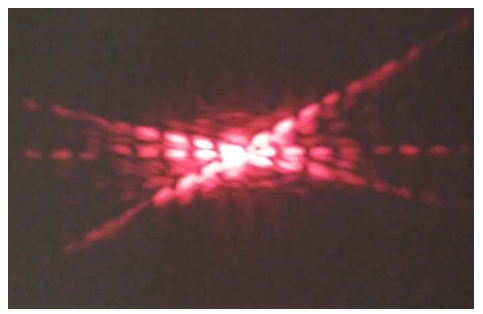

Original (Figure 13)

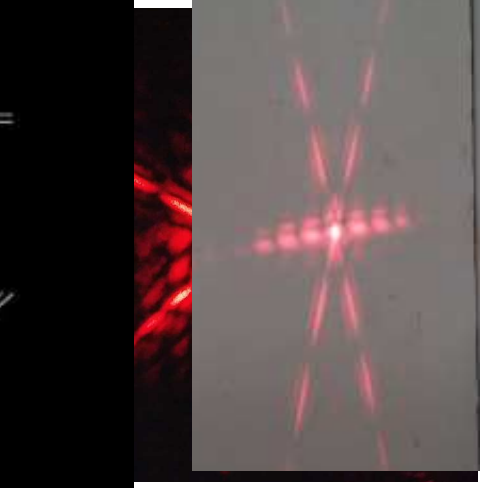

Around Y-axis (Figure 18)

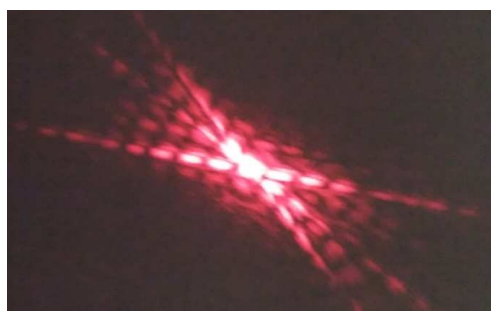

Around X-axis (Figure 21)

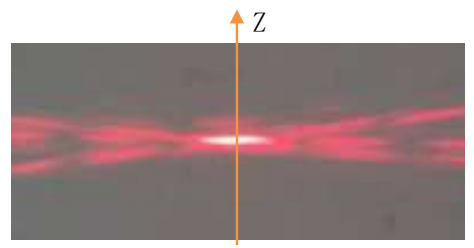

Around Z-axis (Figure 20)

C) Triple-Slit: Rotating around X-axis, Y-axis, Z-axis

With the same diaphragm (Figure 28), comparing Figure 28 (rotating around X-axis), Figure 23/24 (rotating around Y-axis clockwise/counterclockwise) and Figure 27 (rotating around Z-axis), we conclude that which axis the diaphragm rotates around determines the photon's behavior and pattern in the triple slit experiments.
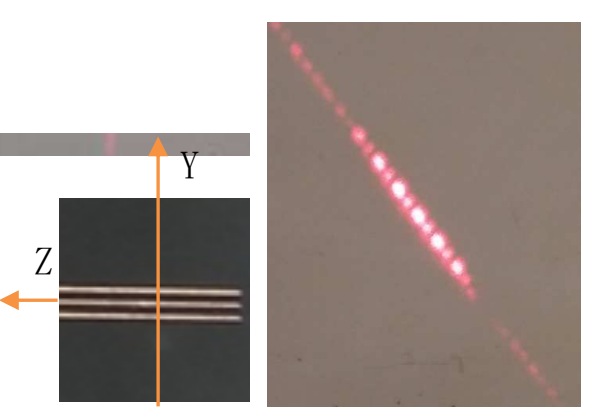

Around X-axis (Fig. 28)
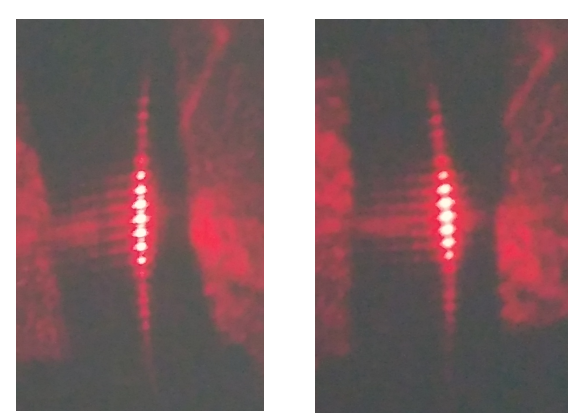

Around Y-axis (Figs. 23 And 24)
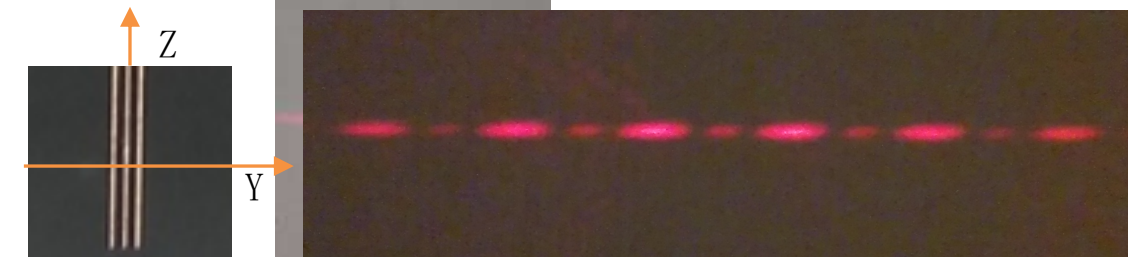

Around $\mathrm{Z}$ axis (Fig. 27)

D) Single Slit: Rotating around X-axis, Y-axis, Z-axis 
With the same diaphragm (Figure 33), comparing Figure 36 (rotating around X-axis), Figure 31/32 (rotating around Y-axis clockwise/counterclockwise) and Figure 35 (rotating around Z-axis), we conclude that which axis the diaphragm rotates around determines the photon's behavior and pattern in the single slit experiments.
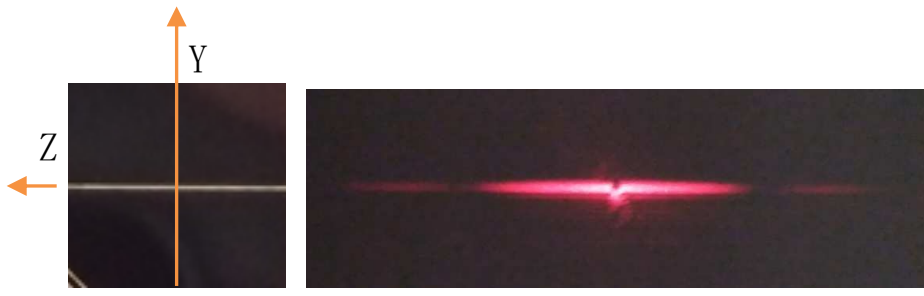

Original (Fig. 33)

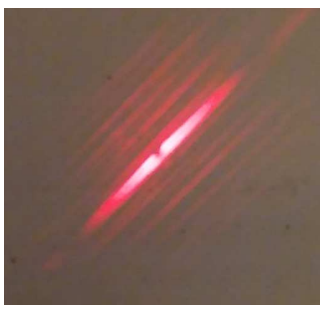

Around X-axis (Fig. 36)
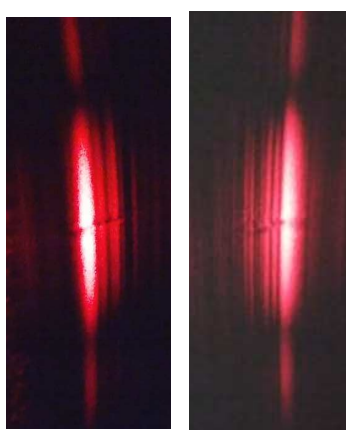

Around Y-axis (Fig. 31/32)

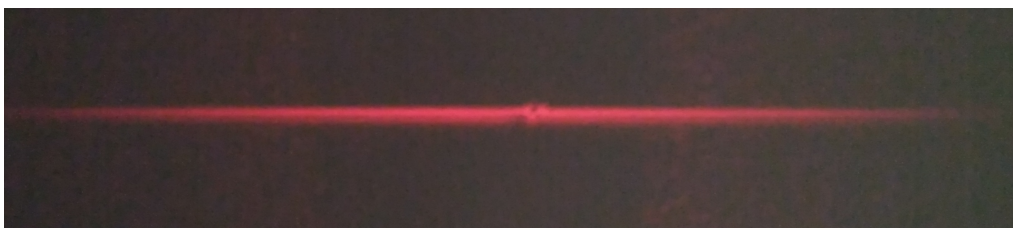

Around Z-axis (Fig. 35)

The above experiments show novel phenomena of the double slit/cross-double slit/triple slit/single slit experiments

\subsection{Comparing Experiments of Different Diaphragms Rotating Around Same Axis}

\section{A) Rotating around $\mathrm{X}$-axis: rotation-symmetry}

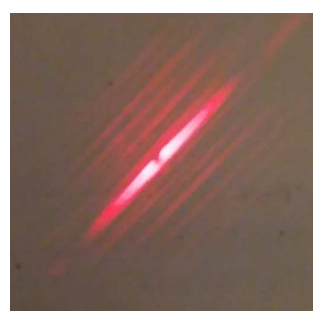

Single slit

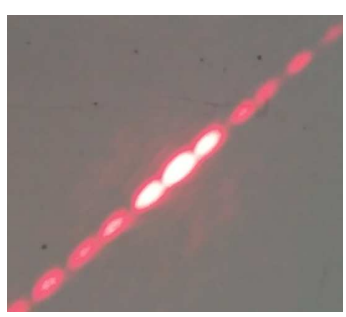

double slit

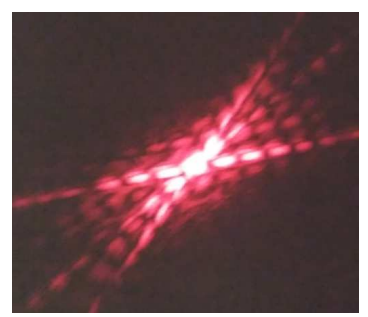

cross-double slit

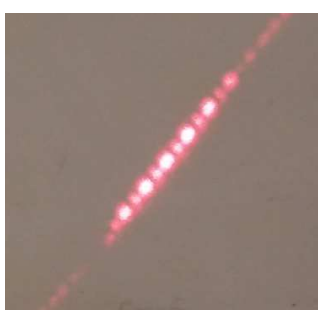

triple slit

B) Rotating around Y-axis: curved pattern 


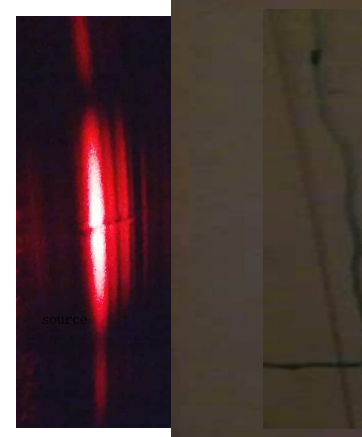

Single slit

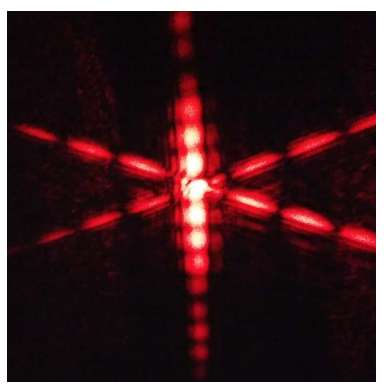

Cross-double slit

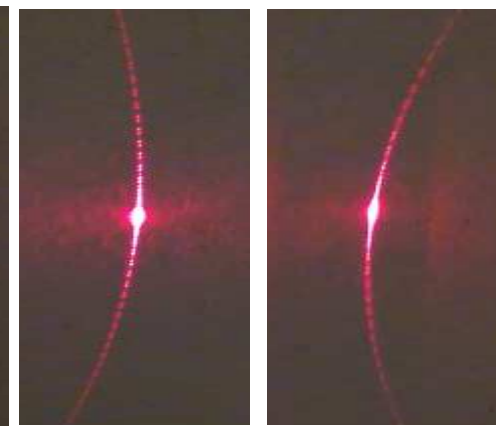

Double slit
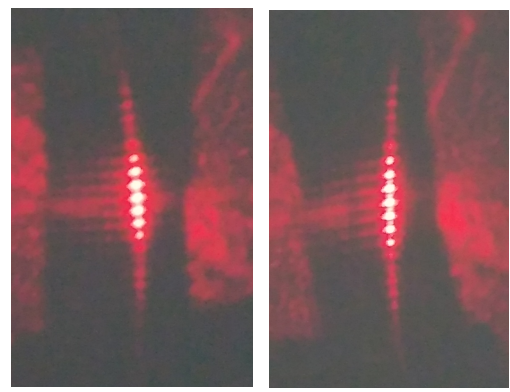

Triple slit

C) Rotating around Z-axis: expansion of patterns
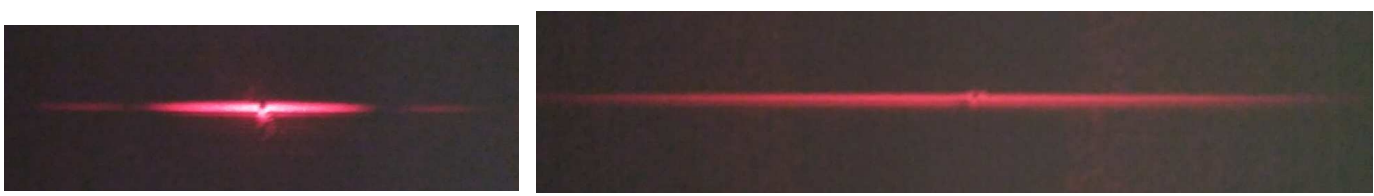

Single slit: original and rotated
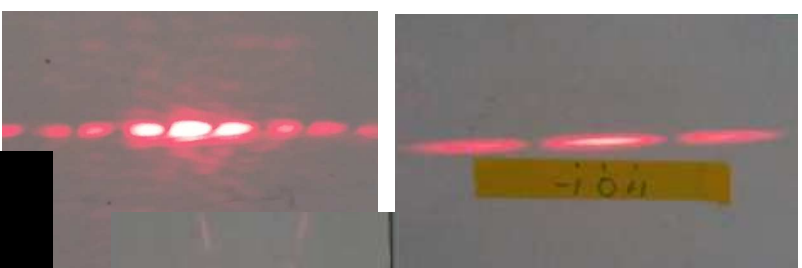

Double slit: original and rotated

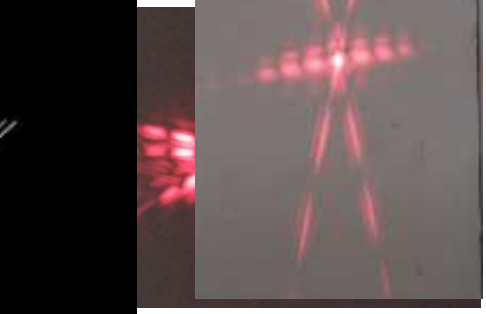

$4 \mathrm{Z}$

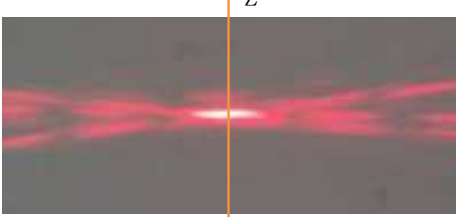

Cross-double slit: original and rotated 


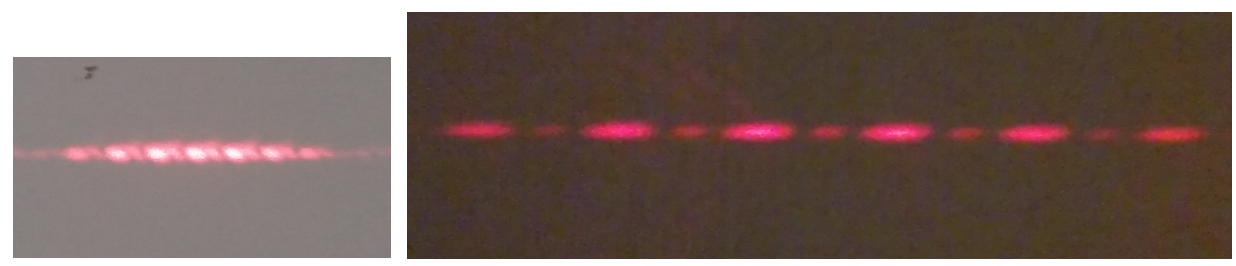

Triple slit: original and rotated

We show the novel phenomena of the double slit/cross-double slit/triple slit/single slit experiments:

(1) unlike the standard single slit/double slit/cross-double slit/triple slit experiments, in which the pattern/interference patterns are along the straight line, the pattern/interference patterns of the single slit/double slit/cross-double slit/triple slit experiments can be along curve, and the curvatures depend on the orientations of the diaphragms and the distance between the diaphragm and screen;

(2)the characteristics of the pattern/interference patterns of the single slit/double slit/cross-double slit/triple slit experiments depend on the orientation of the diaphragm used in the experiment, i.e., the rotation around which axis (either $\mathrm{X}$-axis or $\mathrm{Y}$-axis or Z-axis), the rotation angle (either rotate discrete angles or continuous rotation), the rotation direction around $\mathrm{Y}$-axis (either clockwise or counterclockwise);

(3) in the cross-double slit experiments, the characteristics of the interference patterns also depend on which slit photons passed through.

When rotating around the same axis, the patterns/interference patterns of single slit, double slit, cross-double slit and triple slit experiments show the same phenomena, we call those phenomena "universal phenomena".

Now we have comprehensive phenomena/data to be described and interpreted.

\section{Appendixes}

\section{A1. Video: Evolution of Curved Interference Pattern of Double Slit Experiment}

\section{A2. Potential Applications of Universal Phenomena}

The expansion of the patterns/interference patterns may be utilized in the applications of the double slit, such as the measurement equipment in industry, to improve the accuracy and sensitivity of the measurements.

\section{References}

[1] A. Ananthaswamy, "Through Two Doors at Once”, Dutton, New York, NY, (2018).

[2] A. Robinson, “The Last Man Who Knew Everything”. New York, NY: Pi Press., (2006). 
[3] R. Feynman, R. Leighton, and M. Sands, “The Feynman Lectures on Physics” (Addison-Wesley, Reading, 1966), Vol. 3.

[4] S. Rashkovskiy, Is a rational explanation of wave-particle duality possible? arXiv 1302.6159 [quant-ph] 2013.

[5] Hui Peng, "Novel Double Slit and Cross-Double Slit Experiments: Interference Patterns Depending on Orientations of Diaphragms", Research Square, preprint, DOI: $10.21203 /$ rs.3.rs-653201/v1, 2021.

[6] Hui Peng, "Curved Interference Pattern---Novel Double Slit and Cross-Double Slit experiment (2)”, Research Square, preprint, DOI: https://doi.org/10.21203/rs.3.rs-677223/v1, 2021. 


\section{Supplementary Files}

This is a list of supplementary files associated with this preprint. Click to download.

- 20210630evolutionofcurvedinterferencepattern.mp4 